\title{
The Role of Cellulose Based Separator in Lithium Sulfur Batteries
}

\author{
Nejc Pavlin, ${ }^{1,2}$ Silvo Hribernik, ${ }^{3}$ Gregor Kapun, ${ }^{1}$ Sara Drvarič Talian, ${ }^{1,2}$ Christian Njel,, 4 \\ Rémi Dedryvère, $\mathbb{D}^{4,5}$ and Robert Dominko $\mathbb{(} \mathbb{1}^{1,2,5, \mathrm{z}}$ \\ ${ }^{I}$ National Institute of Chemistry, SI-1000 Ljubljana, Slovenia \\ ${ }^{2}$ University of Ljubljana, Faculty of Chemistry and Chemical Technology, SI-1001 Ljubljana, Slovenia \\ ${ }^{3}$ University of Maribor, Faculty of Mechanical Engineering, 2000 Maribor, Slovenia \\ ${ }^{4}$ CNRS/Univ. Pau \& Pays Adour, Institute of Analytical Sciences and Physical Chemistry for Environment and \\ Materials, IPREM - UMR 5254, 64000 Pau, France \\ ${ }^{5}$ ALISTORE - European Research Institute, Amiens 80039 Cedex, France
}

\begin{abstract}
In this work, abundant and environmentally friendly nano-fibrillated (NFC) cellulose is used for fabrication of porous separator membranes according to the procedure adopted from papermaking industry. As-prepared NFC separators were characterized in terms of thickness, porosity, wettability, electrochemical stability and electrochemical performance in lithium-sulfur and Li-symmetrical pouch cells and compared to a commercial Celgard 2320 separator membrane. Results demonstrated that morphology and electrochemical performance of NFC separator outperforms the conventional polyolefin separator. Due to exceptional interplay between lithium metal and cellulose, this research provides a self-standing NFC separator that can be used besides the lithium-sulfur also in other lithium metal battery configurations.

(C) The Author(s) 2018. Published by ECS. This is an open access article distributed under the terms of the Creative Commons Attribution 4.0 License (CC BY, http://creativecommons.org/licenses/by/4.0/), which permits unrestricted reuse of the work in any medium, provided the original work is properly cited. [DOI: $10.1149 / 2.0401903$ jes]

(cc) BY
\end{abstract}

Manuscript submitted October 3, 2018; revised manuscript received December 17, 2018. Published December 27, 2018. This paper

is part of the JES Focus Issue of Selected Papers from IMLB 2018.

Lithium-sulfur ( $\mathrm{Li}-\mathrm{S})$ batteries are one of the most promising candidates to couple renewable energy sources for green transportation and large-scale energy storage - owing to their various desirable characteristics including competitive cost, and low environmental impact. ${ }^{1,2}$ Moreover, their theoretical gravimetric energy density is much higher than that of conventional $\mathrm{Li}$-ion batteries based on insertion electrodes. Despite these advantages, massive implementation of $\mathrm{Li}-\mathrm{S}$ batteries remains hindered by various challenges concerning capacity fading, predominantly due to loss of active material, nonstable electrochemical interface between lithium metal and different types of electrolytes and high amount of liquid electrolyte used in experiments.

Different approaches have been proposed to mitigate the above mentioned problems. Majority of work was focused on encapsulation of sulfur in different types of meso or micro porous carbon host matrices, but only with partial success. ${ }^{3-5}$ More recently, adsorption additives become attractive due to possible weak bonding of polysulfides with different heteroatoms and structures. ${ }^{6}$ In order to minimize, or even completely avoid dissolution of polysulfides in liquid electrolytes, novel electrolyte formulations are tested with different affinity for polysulfide solubility. Sparingly soluble electrolytes ${ }^{7}$ and fluorinated ethers ${ }^{8-10}$ are showing some promising directions, while partial solubility of polysulfides can still persist as a problem for long term cycling stability. Much less work has been done on separators; i.e. tuning their properties like porosity, tortuosity, compressibility and ion selectivity. ${ }^{11}$ While the first three parameters can be tuned by shape and size of pores, ion selectivity is a function of surface groups. Separator in Li-S batteries is an important part of the battery configuration since besides its traditional role to electronically separate two electrodes, it serves as a reservoir for excess of electrolyte required for Li-S battery operation. Quantity of electrolyte in Li-S batteries plays an important role since higher quantities enable solubilization of intermediate products in form of different lithium polysulfides to a larger extent as well as longer cycle life since electrolyte is constantly consumed for passivation of the fresh lithium surface. ${ }^{12-14}$ Besides that, separator can act as a barrier

${ }^{\mathrm{z} E-m a i l: ~ R o b e r t . D o m i n k o @ k i . s i ~}$ which can prevent or completely stop polysulfide shuttle with proper functionalization. ${ }^{15}$

Separators are considered as a costly part of batteries due to expensive raw materials and complicated fabrication technology. ${ }^{16}$ For that reason, sustainable and cost effective materials are required and should be explored. Among different possibilities, cellulose offers itself as one of the most abundant and renewable resources on Earth and it can be obtained in different forms. Besides that, cellulose possesses outstanding properties such as high dielectric constant, good chemical stability, and superior thermal stability. ${ }^{17}$ Cellulose based separators are considered as electrolyte-philic membranes considerably improving wettability with the electrolyte and with that ionic conductivity between electrodes. ${ }^{18}$ Hydroxyl groups in the structure can be additionally functionalized to adjust surface properties for battery applications. Anisotropic structure of cellulose based separators suppresses lithium dendrite formation, ${ }^{19}$ which was ascribed also to the excellent electrolyte uptake ability and good electrolyte distribution capability on the surface of metallic lithium. ${ }^{20}$ Additionally, by proper selection of the type of cellulose and its structure, separators with desired porosity and thickness can be obtained. Liao et al. described the use of paraffin oil to achieve desirable porosities in cellulose aerogels. ${ }^{21}$ Paraffin oil is incorporated between cellulose fibers and it disables intermolecular bonding of cellulose fibers. Porosities of cellulose membranes can also be tuned by varying the composition ratio of solvent mixture in cellulose dispersion (alcohol/water system).$^{22}$ Beside the use of cellulose for the manufacture of battery separators, the attention of scientists has recently been drawn by the production of all battery components made of cellulosic materials. ${ }^{23,24}$

In this contribution we show electrochemical performance of separators prepared from nanofibrillated cellulose (NFC). NFC separators were prepared by laydown method using dispersion of NFC with paraffin oil stabilized by surfactant. Separators with different loadings of cellulose fibers and thicknesses were tested in the Li-S battery configuration. It was demonstrated that cellulose based separators improve capacity retention in Li-S batteries and much slower metallic lithium anode degradation was observed. The latter was studied by XPS, FIB-SEM and impedance spectroscopy, which confirmed lower degree of lithium degradation as a result of formation of stable passive film enriched with many oxygen moieties. 


\section{Experimental}

Materials. - Nanofibrillated cellulose (NFC) hydrogel $\left(\mathrm{w}_{\text {cellulose }}=\right.$ $3 \mathrm{wt} \%$ ) was supplied from University of Maine, USA. Lithium bis(trifluoromethane) sulfonimide (LiTFSI; 99.0\%), tetraethylene glycol dimethyl ether (TEGDME; 99.0\%), 1,3-dioxolane (DOL, $99.8 \%, 75$ ppm BHT as inhibitor), surfactant Brij C-10, polyvinylidene fluoride (PVdF), multi-walled carbon nanotubes (MWCNT), $\mathrm{N}$-methylpyrrolidone (NMP), $n$-butanol (99.9\%), and LP30 electrolyte (1.0 $\mathrm{M} \mathrm{LiPF}_{6}$ in EC:DMC (1:1, in volume); battery grade) were purchased from Sigma-Aldrich. LiTFSI was additionally dried at $140^{\circ} \mathrm{C}$ in vacuum oven under reduced pressure over night, TEGDME and DOL were additionally dried using molecular sieves, $\mathrm{Na} / \mathrm{K}$ alloy, and distillation. Other chemicals were used without any further purification. Lithium foil (FMC, $110 \mu \mathrm{m}$ ), ENSACO 350G carbon (IMERYS), sulfur (99.5\%, Honeywell), $n$-hexane (95.0\%, Merck), acetone $(99.5 \%$, Merck), and paraffin oil (PharmaChem) were of analytical grade and were used as received without any further purification.

Preparation of nanofibrillated cellulose separators.-Controlled amount of NFC hydrogel was dispersed in $0.2 \mathrm{wt} \%$ Brij C-10 surfactant solution and ultrasonically treated for $1 \mathrm{~h}$ to form $5 \mathrm{mg} \mathrm{mL}^{-1}$ NFC dispersion. Then $1 \mathrm{~mL}$ of paraffin oil (PO) per $100 \mathrm{~mL}$ of cellulose dispersion was added and dispersion was stirred vigorously for $3 \mathrm{~h}$ to obtain homogeneous NFC/PO dispersion. Different amounts of as-prepared dispersion were casted on the polyethylene petri dishes and dried at $50^{\circ} \mathrm{C}$ to remove solvent in order to obtain membranes with different thicknesses. Membranes were then punched into circular discs with a diameter of $18 \mathrm{~mm}$ and placed in hexane to extract paraffin oil and surfactant. After extraction, membranes were washed thoroughly with acetone and ethanol and dried in vacuum oven under reduced pressure at $180^{\circ} \mathrm{C}$ for 4 days before characterization. For the measurements of thicknesses of prepared membranes we used Garant external micrometer (DIN 863). The final thicknesses of NFC separators varied from 31 to $64 \mu \mathrm{m}$. A commercial separator (Celgard 2320; $\mathrm{PP} / \mathrm{PE} / \mathrm{PP}$, thickness $=20 \mu \mathrm{m}$ ) was chosen as a control sample.

\section{Characterization}

Characterization of separator membranes.-The surface morphologies of NFC membranes were analyzed using a field emission scanning electron microscope (FE SEM; Supra 35 VP, Carl Zeiss). Samples were sputter coated with platinum (Pt, $80 \mathrm{~s}, 6 \mathrm{~nm}$; Gatan 682 Precision Etching and Coating System (PECS)) and exposed to an accelerating voltage of $1.5 \mathrm{kV}$.

The porosity of separators was estimated by immersing them in $n$-butanol for $1 \mathrm{~h}$. Separator porosities were then calculated using the equation:

$$
\text { Porosity }=\left(m_{\mathrm{W}}-m_{\mathrm{D}}\right) / \rho_{\mathrm{B}} * V_{\mathrm{M}} * 100 \%,
$$

where $m_{\mathrm{W}}$ is the weight of fully soaked membrane, $m_{\mathrm{D}}$ is the weight of dry membrane, $\rho_{\mathrm{B}}$ is the density of $n$-butanol, and $V_{\mathrm{M}}$ is the apparent volume of the membrane sample.

To assess the wettability of separators, contact angle between separators and liquid electrolyte $\left(1.0 \mathrm{~mol} \mathrm{~L}^{-1}\right.$ LiTFSI in a solvent mixture of TEGDME and DOL (1:1, in volume)) were measured using Theta Optical Tensiometer.

To investigate water content in cellulose, NFC membranes were first dried at $60^{\circ} \mathrm{C}$ in vacuum oven over night, and then transferred in Ar filled dry box $\left(\mathrm{O}_{2}<1.0 \mathrm{ppm}, \mathrm{H}_{2} \mathrm{O}<1.0 \mathrm{ppm}\right)$. Approximately $2 \mathrm{mg}$ of NFC membranes were accurately weighed, put in a glass vial, and dried in vacuum oven inside the dry box at different temperatures $\left(105^{\circ} \mathrm{C}\right.$ and $\left.180^{\circ} \mathrm{C}\right)$ for different time periods. After drying, $2 \mathrm{~mL}$ of 1,3-dioxolane (ultra-dry; $\mathrm{H}_{2} \mathrm{O}$ content $<1.0 \mathrm{ppm}$ ) were added to each NFC sample. Samples were incubated for a week inside the dry box. Water in 1,3-dioxolane was determined using coulometric Karl-Fisher titration.
To evaluate the thermal properties of the nano-fibrillated cellulose separator membranes, thermal analyser (NETZSCH STA 449F3) was used. Samples were scanned from $40^{\circ} \mathrm{C}$ to $900^{\circ} \mathrm{C}$ at a heating rate of $10^{\circ} \mathrm{C} /$ min under $\mathrm{Ar}$ atmosphere.

Post-mortem characterization of lithium metal electrodes.-XPS analyses were performed with a Kratos Axis Ultra-spectrometer using focused monochromatized $\mathrm{Al} \mathrm{K} \alpha$ radiation $(\mathrm{h} \nu=1486.6 \mathrm{eV})$. To prevent exposure of lithium electrodes to moisture/air, all samples were packed and stored in argon-filled dry box. Before XPS analysis, all samples were washed with DOL (less than $0.4 \mathrm{ppm}$ of water content), to reduce the amount of salt on the surface of the sample. The spectrometer was calibrated using the $\mathrm{Ag} 3 \mathrm{~d}_{5 / 2}$ photoemission peak at $368.3 \mathrm{eV}$ with a full width at half-maximum (FWHM) of $0.58 \mathrm{eV}$ and each photoemission spectrum was recorded with constant pass energy of $20 \mathrm{eV}$. The pressure in the analysis chamber was around $5 \times 10^{-9}$ mbar and the analyzed area of the samples was $300 \times 700 \mu \mathrm{m}^{2}$. Short-scan spectra were measured before and after the usual longscan experiment in order to check for possible degradation of lithium samples due to X-ray exposure. The binding energies were calibrated from the hydrocarbon contamination using the $\mathrm{C} 1 \mathrm{~s}$ peak at $285 \mathrm{eV}$. The peaks were quantified on the basis of Scofield's relative sensitivity factors.

Focused ion beam - scanning electron microscope (FIB - SEM Helios Nanolab 650i) was used for post-mortem cross - sectional analysis of lithium metal electrodes. The surface of lithium electrode was first protected with in situ deposited platinum to prevent a curtaining effect. Platinum on lithium samples was deposited in two steps. In the first step, the electron-beam assisted platinum deposition was used. The deposition was performed at $2 \mathrm{kV}$ of accelerating voltage and at a current of $0.4 \mathrm{nA}$ for $30 \mathrm{~min}$. The thickness of deposited layer was around $300 \mathrm{~nm}$. In second step, faster ion-beam was used to deposit the remaining thickness. The deposition was achieved at $30 \mathrm{kV}$ of accelerating voltage and at current of $0.43 \mathrm{nA}$ for $20 \mathrm{~min}$. The thickness of deposited layer was around $500 \mathrm{~nm}$. All samples were prepared in an argon-filled dry box and transferred into the microscope under argon atmosphere.

Electrochemical characterization.-The sulfur electrodes for electrochemical measurements were prepared by conventional slurrycasting method with a doctor blade applicator. Sulfur/ENSACO 350G composite was obtained by ball milling sulfur (66.7 wt\%) and ENSACO $350 \mathrm{G}$ carbon $(33.3 \mathrm{wt} \%)$ at $300 \mathrm{rpm}$ for $30 \mathrm{~min}$. So prepared mixture was consequently heated to $155^{\circ} \mathrm{C}$ for $5 \mathrm{~h}$ with a $0.2^{\circ} \mathrm{C} \mathrm{min}-1$ heating ramp under argon flow. The cathode slurry was prepared by mixing $80 \mathrm{wt} \%$ sulfur/ENSACO 350G carbon composite, $10 \mathrm{wt} \%$ MWCNT, and $10 \mathrm{wt} \% \mathrm{PVdF}$ binder in NMP with ball milling (30 $\min , 300 \mathrm{rpm})$. The slurry was then cast onto carbon coated aluminum foil using doctor blade $(250 \mu \mathrm{m})$ and dried at $50^{\circ} \mathrm{C}$ overnight. The cathodes were then punched into round discs with a diameter of $14 \mathrm{~mm}$ and stored in an argon filled dry box before use. The sulfur loading varied from 1.0 to $1.5 \mathrm{mg} \mathrm{cm}^{-2}$.

In order to investigate electrochemical stability of NFC separator, cyclic voltammetry experiment was performed on a working sulfur electrode and a counter lithium foil electrode at a scan rate of $0.1 \mathrm{mV} \mathrm{s}^{-1}$. Further evaluation was carried out using different electrolytes and cyclic voltammetry experiment performed on a stainlesssteel working electrode with lithium counter electrode at a scan rate of $0.1 \mathrm{mV} \mathrm{s}^{-1}$. To examine Li metal stability during Li stripping/ deposition, Li|separator|Li cells with two different separators were assembled. Tests were conducted at a current density of $0.5 \mathrm{~mA} \mathrm{~cm}{ }^{-2}$, using $20 \mu \mathrm{L}$ of electrolyte. The first two cycles were $4 \mathrm{~h}$ long (areal capacity of $2 \mathrm{mAh} \mathrm{cm}^{-2}$ ), all the others cycles lasted for $2 \mathrm{~h}$ (areal capacity of $1 \mathrm{mAh} \mathrm{cm}^{-2}$ ).

Alternating current (AC) impedance measurements (VMP3, Bio-Logic) were done on Li symmetrical cells. After assembly of the cells, they were left for $24 \mathrm{~h}$ in order to stabilize the passive layer growth. During this time, EIS measurements in the range of $1 \mathrm{MHz}$ to $1 \mathrm{mHz}$ were measured. After determination of EIS contributions 


\begin{tabular}{|c|c|c|c|}
\hline Sample & $\begin{array}{l}\text { Real mass } \\
{\left[\mathrm{mg} \mathrm{cm}^{-2}\right]}\end{array}$ & Thickness $[\mu \mathrm{m}]$ & Porosity [\%] \\
\hline NFC_15PO & $1.50 \pm 0.04$ & $33 \pm 2$ & $44 \pm 2$ \\
\hline NFC_20PO & $1.84 \pm 0.06$ & $42 \pm 1$ & $47 \pm 1$ \\
\hline NFC_25PO & $2.38 \pm 0.05$ & $49 \pm 2$ & $51 \pm 1$ \\
\hline NFC_30PO & $2.98 \pm 0.06$ & $62 \pm 2$ & $57 \pm 1$ \\
\hline NFC_20 & $2.0 \pm 0.2$ & $28 \pm 3$ & $6.0 \pm 0.5$ \\
\hline Celgard 2320 & $1.14 \pm 0.04$ & $20.0 \pm 0.4$ & $42 \pm 3$ \\
\hline
\end{tabular}

on fresh stabilized cells, the experiments were continued at different steps during the stripping and deposition experiment. After each cycle of stripping and deposition, the current was stopped for 15 minutes and EIS spectra were measured with $10 \mathrm{mV}$ (RMS) amplitude in the frequency range from $1 \mathrm{MHz}$ to $1 \mathrm{mHz}$. To ensure comparable diffusional lengths, two Celgard 2320 separators were used in the cell employed for comparison.

To evaluate cycle performance of separators in the $\mathrm{Li}-\mathrm{S}$ batteries, pouch-bag cells were assembled. The cells were cycled galvanostatically between 3.0 and $1.5 \mathrm{~V}$ at a fixed charge/discharge current density of $0.1 \mathrm{C}\left(1 \mathrm{C}=1672 \mathrm{~mA} \mathrm{~g}^{-1}\right)$ (MPG-2, Bio-Logic). The electrolyte quantity was normalized to $20 \mu \mathrm{L} \mathrm{mg}^{-1}$ of sulfur. To explore $\mathrm{C}$ rate capability, discharge current densities were varied from $0.05 \mathrm{C}$ to $1 \mathrm{C}$ (MPG-2, Bio-Logic).

All battery cells were assembled in an argon-filled dry box $\left(\mathrm{O}_{2}<\right.$ $\left.1.0 \mathrm{ppm}, \mathrm{H}_{2} \mathrm{O}<1.0 \mathrm{ppm}\right)$. Sulfur cathode and lithium foil $(14 \mathrm{~mm}$ in diameter) were separated with Celgard 2320 or NFC membrane which was wetted with 1.0 M LiTFSI TEGDME:DOL (1:1, in volume) electrolyte.

\section{Results and Discussion}

Nanofibrillated cellulose (NFC) based separators were prepared by laydown method, imitating papermaking process, by using water dispersion with paraffin oil (PO) as a pore-forming agent and Brij $\mathrm{C}-10$ as a surfactant. A homogenous and stable dispersion was prepared and used for preparation of samples in the form of thin sheets with a uniform thickness (from 28 to $62 \mu \mathrm{m}$ ). Table I summarizes properties (mass, thickness and porosity) of four prepared NFC samples and compares them with a selected Celgard 2320 separator. Separators prepared by addition of paraffin oil are denoted as NFC_xyPO, whereas separator prepared without PO additive is denoted as NFC_20. By increasing the mass loading, thickness and porosity of cellulose separator increases. Latter can be attributed to the anisotropy of long cellulose fibers which increase disorder inside the sheet. It is important to stress that an increase of porosity has been observed only in the case when PO was added into the dispersion of NFC and surfactant. Obtained separator sheets without adding PO (NFC_20) is much denser since for similar loading we obtained a much thinner sheet with almost no porosity. That proves the active role of PO addition during fabrication of separator sheets which helps in the formation of the interconnected pores within the anisotropically interconnected NFC fibers. ${ }^{21}$ Obtained porosity of NFC membranes is comparable with Celgard separator (Table I) while thickness is higher and that has to be further optimized in order to obtain even thinner and uniform separators. Nevertheless, to the best of our knowledge the reported thicknesses of self-standing NFC membranes in this study are one of the thinnest among those reported in the literature. ${ }^{17,22,25} \mathrm{In}$ terestingly, masses of the Celgard and the thinnest separator prepared in our study are very similar, although the cellulose based separator is thicker. Top down views of NFC_20PO (separator prepared with adding PO and surfactant) and NFC_20 (separator prepared without any additive in water dispersion) are shown in Figure 1. NFC_20PO (Figure 1a) separator shows open pore structure with interconnected pores in diameter between $100 \mathrm{~nm}$ to $1 \mu \mathrm{m}$. Addition of PO and
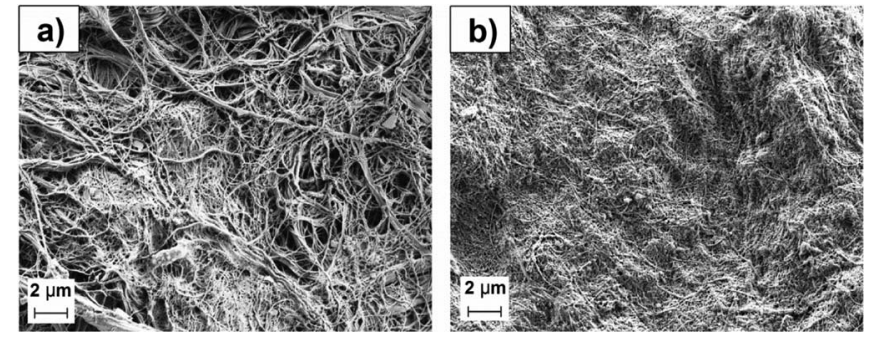

Figure 1. Morphology of the surface of separators prepared from water dispersion: a) containing NFC fiber, PO, and surfactant and b) containing only NFC fibers.

surfactant enables formation of pores, however, some parts are still partially closed which implies that preparation procedure is still not fully optimized. On the other hand, the separator prepared without additives (Figure 1b) shows a much more closed, densely packed surface, without any visible macro pore structure. NFC fibers tend to compactly collapse by capillary action during the evaporation of water, and are fixed with strong hydrogen bonds of cellulose chains, thus yielding dense-structured membranes. ${ }^{26}$

Cellulose is a natural polymer with high affinity for water, meaning that its drying procedure is highly important for application in batteries. In our work we have developed a standard procedure in which NFC fibers are dried for at least $72 \mathrm{~h}$ at $180^{\circ} \mathrm{C}$. In this way we managed to reduce content of water in the NFC fibers below $0.2 \mathrm{wt} \%$ (Figure S1). The final content of water in the NFC fibers would at the end correspond to an increase of water content within the range of few ppm.

Thermal stability of the separator is another crucial aspect in battery safety. ${ }^{27}$ According to the TGA analysis shown in Figure S2, the $\mathrm{NFC}$ separator possesses superior thermal stability up to $250^{\circ} \mathrm{C}$. Melting temperature for $\mathrm{PE}$ and $\mathrm{PP}$ is depended on the molecular weight of the polymer (PE melting point $120-130^{\circ} \mathrm{C}$, PP melting point 165 $\left.170^{\circ} \mathrm{C}\right)^{28,29}$ and it is lower than that for NFC membrane. From $100^{\circ} \mathrm{C}$ to $250^{\circ} \mathrm{C}$ small weight loss $(\approx 1 \%)$ is observed due to evaporation of trapped water inside the cellulose structure. This implied that NFC separator possessed better thermal stability than PP/PE/PP separator. Therefore, NFC separator has great potential in battery cells operating at an elevated temperature.

As-prepared porous NFC membranes were cut to circular separators with a diameter of $18 \mathrm{~mm}$ and used in batteries without any other separator. To investigate electrochemical stability of NFC separator in Li-S battery cell, cyclic voltammetry (CV) was performed (Figure $\mathrm{S} 3$ ). The $\mathrm{CV}$ shows no presence of additional cathodic or anodic peaks when NFC separator is used. NFC separator is electrochemically stable in the potential window from 1.5 to $3.0 \mathrm{~V}$ and thus, suitable for use in $\mathrm{Li}-\mathrm{S}$ batteries. Moreover, $\mathrm{CV}$ curves show that NFC separator is electrochemically stable in $1.0 \mathrm{M}$ LiTFSI TEGDME:DOL $(1: 1$, in volume) in potential window from $0 \mathrm{~V}$ to $3 \mathrm{~V}$ and in $1.0 \mathrm{M} \mathrm{LiPF}_{6}$ in EC:DMC (1:1, in volume) in potential window from $0 \mathrm{~V}$ to $5 \mathrm{~V}$, and thus also suitable for other lithium metal batteries (Figure S4).

Figure 2a compares electrochemical performance of $\mathrm{Li}-\mathrm{S}$ batteries using NFC separators with different thicknesses and Celgard 2320 separator. In general, there is no remarkable difference in the $\mathrm{Li}-\mathrm{S}$ batteries electrochemical behavior, except in the initial cycles, which can be ascribed to the higher amount of electrolyte stored in the NFC based membranes. Observed Coulombic efficiencies in the first cycle are lower for all three NFC based separators compared to the $\mathrm{Li}-\mathrm{S}$ cell with Celgard separator. This can be connected with local amount of electrolyte since due to higher pore volume the thickest separator has higher local amount of electrolyte which enables higher absolute quantity of soluble sulfur species (elemental sulfur or polysulfides). Pore volume of the thinnest NFC membrane is close to Celgard separator and first cycle Coulombic efficiency is very close for both Li-S batteries with NFC_15PO and Celgard separators. This result suggest that local amount of electrolyte plays important role in the formation 

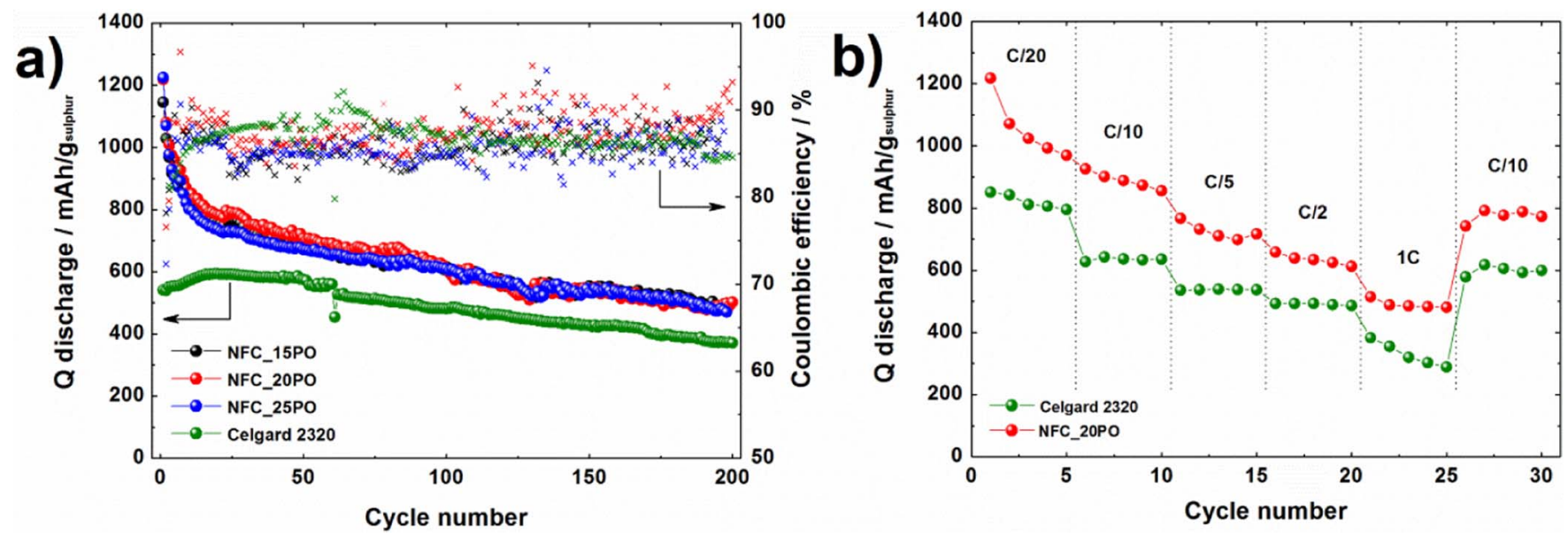

Figure 2. Electrochemical performance of Li-S batteries with different types of porous NFC separators compared to Li-S battery with Celgard separator a) cycling performance and b) rate capability performance (performances of batteries with Celgard and NFC_20 are presented).

cycles while latter on it has almost no impact on cyclability of Li-S batteries, if cell construction enables that electrolyte which cannot be accommodated in the separator is in the vicinity of electrodes. Additionally, higher absolute solubility of sulfur and polysulfides in the locally available electrolyte enables higher initial capacity due to higher ratio between sulfur and electrolyte. Unfortunately that leads to faster capacity fading in the initial 20 cycles. After initial cycles, the performance of all $\mathrm{Li}-\mathrm{S}$ batteries is stabilized and shows slow capacity fading. Nevertheless, $\mathrm{Li}-\mathrm{S}$ batteries with NFC separator show $20 \%$ higher capacity retention within the similar $\mathrm{Li}-\mathrm{S}$ battery configuration which is only different in the separator choice. Similar difference is observed in the rate capability test (Figure 2b). A reason for improved capacity retention could be higher amount of locally available electrolyte. One has to consider that wettability of cellulose fibers with electrolyte is much better and that enables better contact between separator and composite cathode leading to better conversion of polysulfides that were diffused out from the composite cathode. ${ }^{30}$ The wettability of NFC membrane was checked and compared with the Celgard separator. Test showed that wettability angles are 53.9 for Celgard separator, $32.6^{\circ}$ for NFC_20 membrane, and $6.7^{\circ}$ for NFC_20PO membrane (Figure S5).

Another beneficial aspect of cellulose based separators has been recently reported by $\mathrm{R}$. Pan et al. ${ }^{20}$ who explained reduced formation of high surface area lithium (HSAL) due to better wettability of the lithium metal surface when cellulose based separator is used.
Using NFC separators prevents fast degradation of lithium metal and anisotropic nature of nano fibers enables use of self-standing cellulose based separators. In all our tests $(\mathrm{Li}-\mathrm{S}$ batteries and $\mathrm{Li}-\mathrm{Li}$ symmetrical cells) we did not observe electrochemical signatures in the curves characteristic for formation of dendrites. Figure 3 shows stripping and deposition of lithium in the symmetrical cell. In the initial cycles, overpotential required for stripping and deposition of lithium is higher in the case of using NFC separator. This is most probably due to formation of SEI through the reaction between metallic lithium and cellulose (or residual water in cellulose) and due to creation of ionic paths through the SEI formed between metallic lithium and NFC separator. After the initial cycles, overpotential monotonously increases in the first $200 \mathrm{~h}$ and after which the increase in the battery cycled with Celgard separator is exponential, while in the battery with NFC_20PO remains almost constant for $500 \mathrm{~h}$. The observed difference in the overpotential increase during stripping and deposition process is connected with the restructuring of lithium surface and formation of HSAL. In each cycle, a part of lithium is exposed to the electrolyte and needs to be protected by fresh passive film. Due to constant passivation, electrolyte quantity in the testing cell is highly important since in each cycle a small amount of electrolyte is consumed leading to cell dry out. ${ }^{31}$ Another process is formation of HSAL and potential dendrite formation causing safety issues. Differences in the polarization observed in the Figure $3 \mathrm{a}$ are visualized in Figure $3 \mathrm{~b}$ and Figure S6, where SEM micrographs of pristine lithium
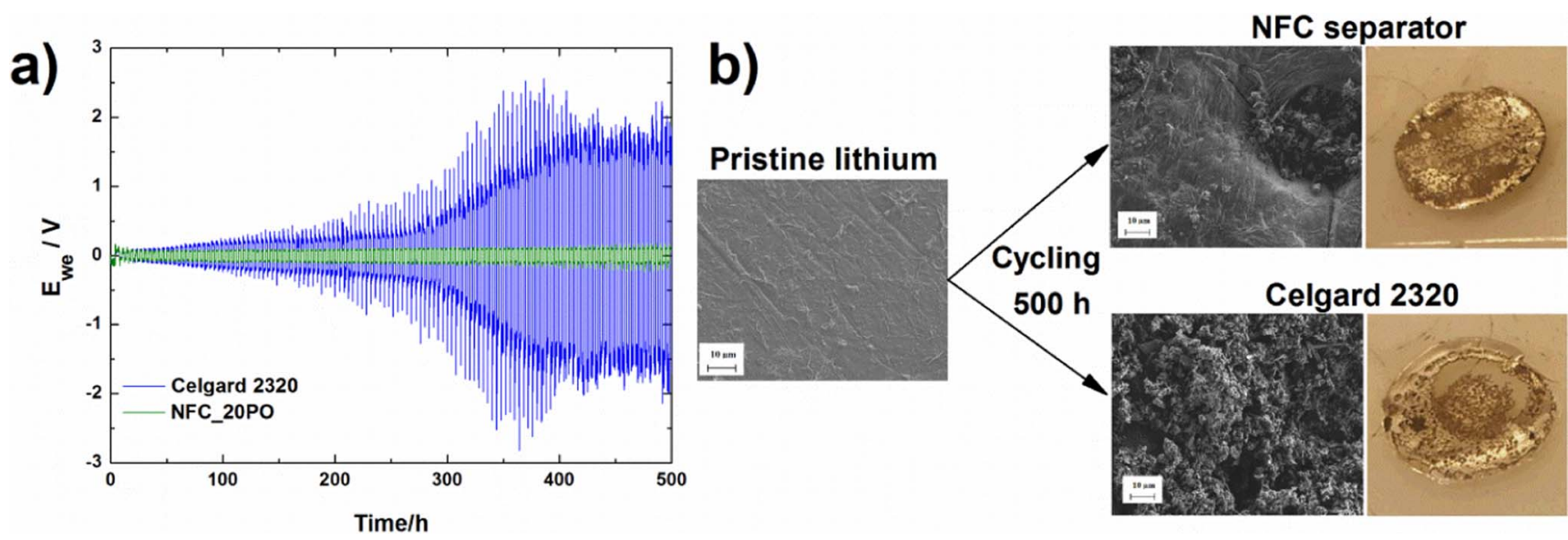

Figure 3. Comparison of the stripping and deposition in the symmetrical Li||Li cells employing NFC_20PO and Celgard separators; a) continuous time limited stripping and deposition profiles at constant current; b) characteristic morphology of lithium before cycling and after $500 \mathrm{~h}$ of cycling for WE faced to NFC_20PO or Celgard separators. 

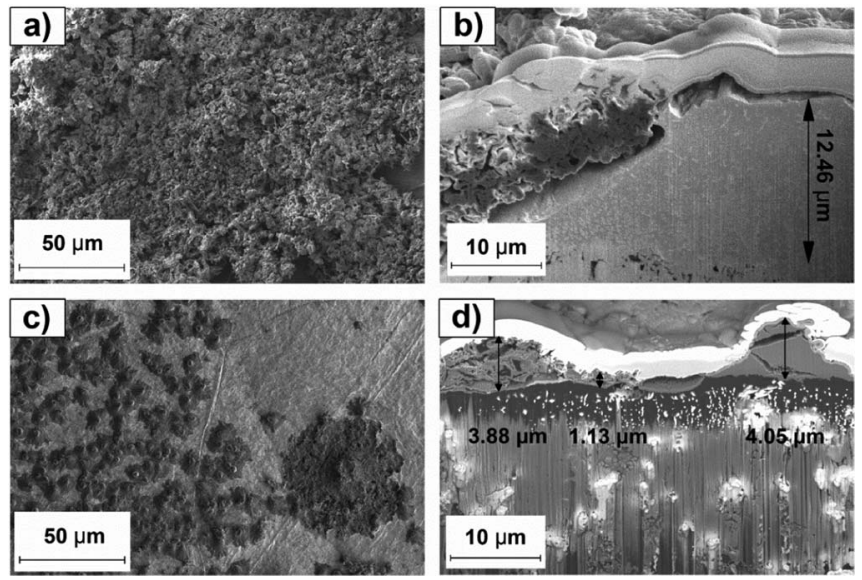

Figure 4. FIB-SEM pictures of lithium anode morphology after cycling for $500 \mathrm{~h}$; a) surface of lithium WE faced to Celgard 2320 separator, b) crosssection of lithium WE faced to Celgard 2320 separator; c) surface of lithium WE faced to NFC separator; d) cross-section of lithium WE faced to NFC separator.

surface and post mortem lithium surfaces after $200 \mathrm{~h}$ and $500 \mathrm{~h}$ of cycling are shown. Electrodes, which served as working electrodes, i.e. electrodes where in the first cycle stripping of lithium was initially started, show remarkable difference in the surface morphology. Most of the surface which was faced to the NFC separator is smooth; almost without any formation of HSAL as observed in the upper-right corner. Visual inspection suggests that lithium dendrites are not intergrown into the NFC based separator. Quite opposite is the morphology of lithium electrode which was faced to the Celgard separator. Visually the surface of lithium anode was corroded and from SEM micrograph we can see that it is completely covered with HSAL, typical for non-homogenous lithium deposition onto conductive surface.

Depth profile of lithium morphology after cycling for $500 \mathrm{~h}$ was checked with FIB-SEM (Figure 4). Similar as observed in the Figure 3, top down view obtained by FIB-SEM (Figures 4a and 4c) shows a difference in the morphology of the deposited lithium. The morphology of lithium deposited in the presence of the Celgard separator is very rough (Figure 4a) confirming mainly formation of HSAL, while the morphology of lithium deposited in the presence of NFC separator (Figure 4c) is much more homogenous with small islands of HSAL. Cross section view prepared by FIB SEM is shown in the Figures $4 \mathrm{~b}$ and $4 \mathrm{~d}$. As expected and communicated also in our previous work, bulk of non-protected lithium electrode after long cycling becomes nanostructured with many pores. ${ }^{32}$ Figure $4 \mathrm{~b}$ shows cross section of lithium electrode which was cycled for $500 \mathrm{~h}$ in a symmetrical cell with Celgard separator. HSAL can be visualized very deep in the bulk (porous structured lithium was observed deeper than $10 \mu \mathrm{m}$ ). Although lithium cycled in the presence of NFC separator exchanged same amount of charge, only few spots of HSAL can be visualized on the surface and as it can be observed in Figure 4d, the formation of HSAL is shallower. From observed differences we can conclude that there is an obvious difference in the morphology of deposited lithium on the lithium surface in the presence of NFC separator compared to the passivation when polyolefin based separator is used in the symmetrical cell.

To better understand differences leading to remarkable change in the mechanism of lithium surface passivation we performed XPS analysis. Sets of two symmetrical cells for each battery configuration were cycled for $50 \mathrm{~h}$ and for $500 \mathrm{~h}$, disassembled and we analyzed the surface of the working electrode facing Celgard 2320 or NFC separators by XPS. O 1s, F 1s, N 1s and S 2p spectra are shown in Figure 5. Additional spectra (survey spectra, $\mathrm{C} 1 \mathrm{~s}$ and $\mathrm{Li} 1 \mathrm{~s}$ core peaks) are provided in Figure S7. O 1s spectra gather signals coming from all oxygenated species at the surface, including $\mathrm{Li}_{2} \mathrm{CO}_{3}$ at $531.8 \mathrm{eV}$ (already observed at the surface of pristine lithium, see corresponding XPS spectra in
Figure S8) and remaining salt traces (-SO $\mathrm{SO}_{2}$ - groups) at $\sim 533 \mathrm{eV}$. Another $\mathrm{O} 1 \mathrm{~s}$ peak is detected at low binding energy $(528.8 \mathrm{eV})$, which can undoubtedly be assigned to $\mathrm{Li}_{2} \mathrm{O}$. F $1 \mathrm{~s}$ and $\mathrm{N} 1 \mathrm{~s}$ spectra display the same level of information with two peaks, one at high binding energy (688.6 eV for F 1s, $399.6 \mathrm{eV}$ for $\mathrm{N}$ 1s) attributed to remaining LiTFSI salt at the surface, and another one at low binding energy $(685.0 \mathrm{eV}$ for $\mathrm{F} 1 \mathrm{~s}, 397.6 \mathrm{eV}$ for $\mathrm{N} 1 \mathrm{~s}$ ) indicating the presence of degradation species of the salt (fluoride $\mathrm{LiF}$ and nitride $\mathrm{Li}_{3} \mathrm{~N}$, respectively), resulting from salt reduction at the lithium surface. Concerning $S 2 p$ spectra, they have to be fitted with $2 p_{3 / 2}-2 p_{1 / 2}$ doublets separated by $1.2 \mathrm{eV}$ with $2 / 1$ intensity ratio due to spin-orbit coupling. The main doublet with $2 \mathrm{p}_{3 / 2}$ component at $169.1 \mathrm{eV}$ is the signal of LiTFSI $\left(\mathrm{S}^{+\mathrm{VI}}\right.$ oxidation state). The three additional doublets $\left(2 \mathrm{p}_{3 / 2}\right.$ at $167.5 \mathrm{eV}, 163.5 \mathrm{eV}$ and $161.5 \mathrm{eV})$ are attributed to sulfur degradation species from the salt (labelled SDS in Figure 5d). The first one at $167.5 \mathrm{eV}$ corresponds to sulfites $\mathrm{S}^{+\mathrm{IV}}$, the two following at $163.5 \mathrm{eV}$ and $161.5 \mathrm{eV}$ correspond to reduced sulfur species originating from salt reduction at the lithium surface. ${ }^{33,34}$

The evolution of measured amounts of $\mathrm{Li}_{2} \mathrm{O}$ and other salt degradation species $\left(\mathrm{Li}_{3} \mathrm{~N}, \mathrm{LiF}\right.$ and sulfur degradation species) is reported in Figure 5e after 50h and 500h of cycling for the two kinds of separators. The relative amounts of $\mathrm{Li}_{2} \mathrm{O}$ compared to other salt species are higher with NFC separator than with Celgard separator, which allows us to assume that $\mathrm{Li}_{2} \mathrm{O}$ is formed by another mechanism than salt reduction in the case of NFC separator. It can be explained by the reaction between hydroxide groups or trapped water in the cellulose fibers and the metallic lithium surface, leading to higher amount of $\mathrm{Li}_{2} \mathrm{O}$ at the surface. For NFC separator, the amounts of $\mathrm{Li}_{2} \mathrm{O}$ and other salt degradation species are high after $50 \mathrm{~h}$ of cycling but decrease after prolonged cycling $\left(500 \mathrm{~h}\right.$ ), while for Celgard, the amounts of $\mathrm{Li}_{2} \mathrm{O}$ and other salt degradation species are much lower after $50 \mathrm{~h}$ of cycling but increase significantly after $500 \mathrm{~h}$. This allows us to assume that these species observed at the surface of lithium are mainly due to the initial reaction of the lithium surface with the separator in the case of NFC, while they are mainly due to Li stripping/deposition process during cycling for Celgard.

Based on the changes of the surface morphology (formation of HSAL) and the amounts of $\mathrm{Li}_{2} \mathrm{O}$ and other salt degradation species, we propose that $\mathrm{Li}_{2} \mathrm{O}$ participates in the passivation of the surface of lithium as an effective electronic insulator. Fresh lithium cannot be deposited directly on the surface due to more dense surface coverage with $\mathrm{Li}_{2} \mathrm{O}$ and it has to diffuse below passive film. ${ }^{35}$ Such mechanism also explains slightly higher polarization in the formation cycles although one would expect lower polarization due to better wettability of the electrodes with electrolyte. Opposite to the formation of the passive film on the lithium in the presence of NFC separator is formation of passive film in the presence of Celgard separator. Here the quantity of detected $\mathrm{Li}_{2} \mathrm{O}$ is lower and consequently also the coverage density is not complete, what influences formation of good ionically conductive and electronically resistive passive film. Fresh, non-protected, electronically conductive surface can serve as an active surface for growth of HSAL which needs to be passivated leading to constant consumption of the electrolyte. Since the quantity of electrolyte is constant that leads to battery dry out and consequently to higher polarization during cycling.

In order to understand differences in the polarization we have performed a systematic electrochemical impedance spectroscopy (EIS) study (Figure 6). Before stripping and deposition, the cells were first left to stabilize for a period of $48 \mathrm{~h}$. EIS spectra during this stabilization are shown in Figure S9. After 48 h, stripping and deposition experiment was conducted in such a way, so that after each cycle the cell impedance spectrum was measured. As already mentioned in the stripping and deposition experiment analysis, higher initial overpotential (also evident in Figures $6 \mathrm{a}$ and $6 \mathrm{c}$, black curve) required in the case of NFC separator is attributed to the formation of passive film. This deduction is supported by the comparison of cell impedance before cycling (Figures $6 \mathrm{~b}$ and $6 \mathrm{~d}$, black spectra), since the high frequency arc (peak frequency 250 to $500 \mathrm{~Hz}$ ) corresponding to the migration of $\mathrm{Li}^{+}$through the SEI is larger with NFC separator $(300 \Omega)$ than with 
a)

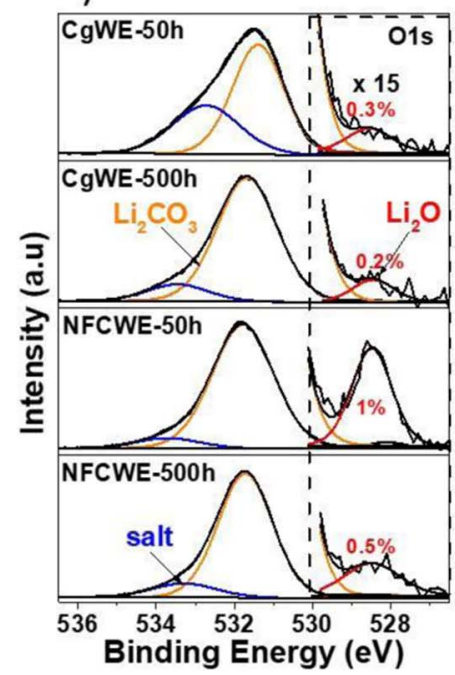

b)

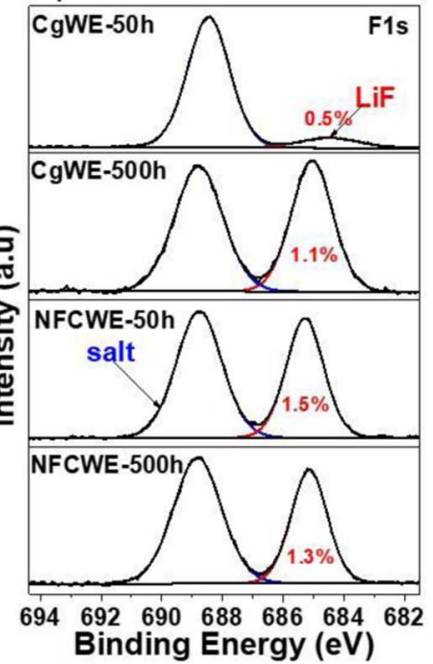

c)

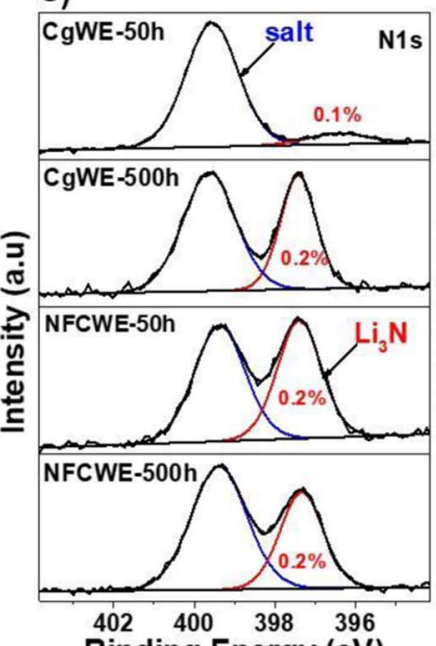

Binding Energy (eV) d)

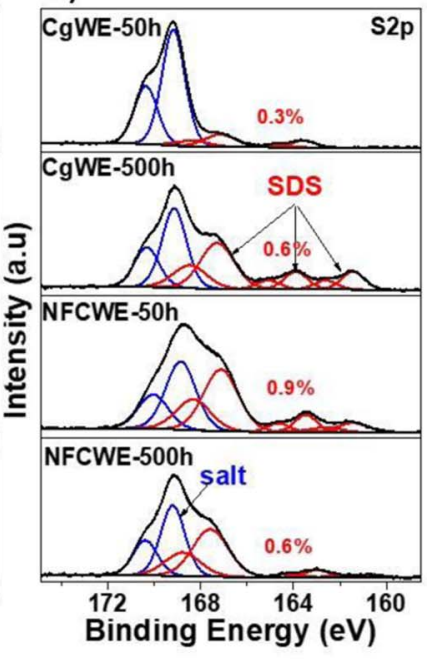

e)

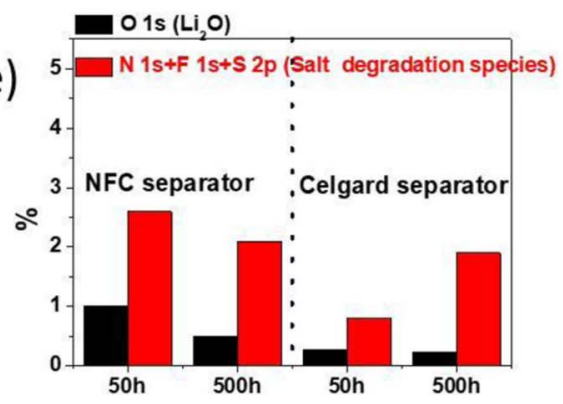

Figure 5. (a) O 1s, (b) F 1s, (c) N 1s and (d) S 2p XPS spectra of Li working electrodes (WE) facing Celgard 2320 or NFC separator, after 50 and $500 \mathrm{~h}$ of cycling. (e) Amounts of $\mathrm{Li}_{2} \mathrm{O}$ and salt degradation species. ( $\mathrm{SDS}=$ sulfur degradation species).
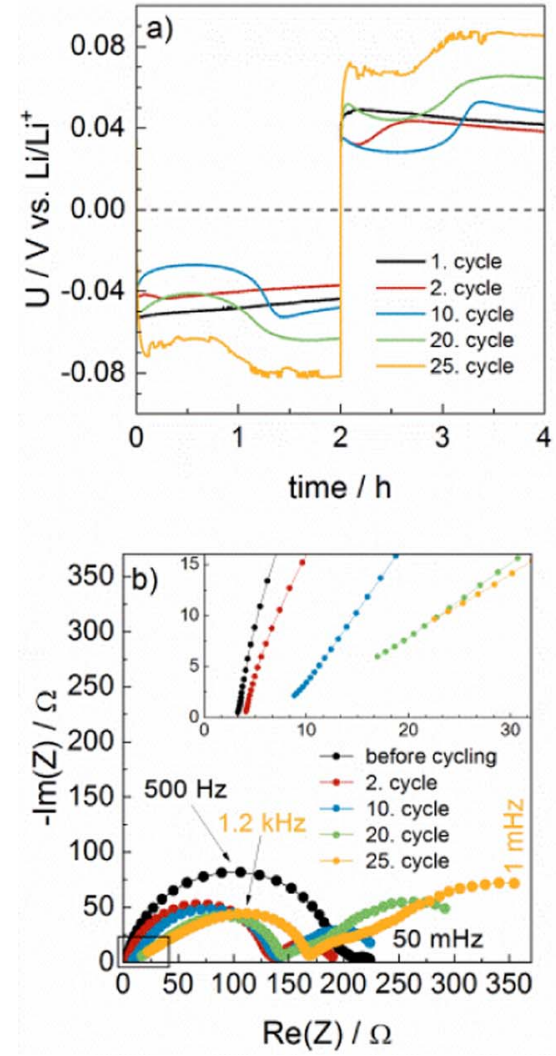
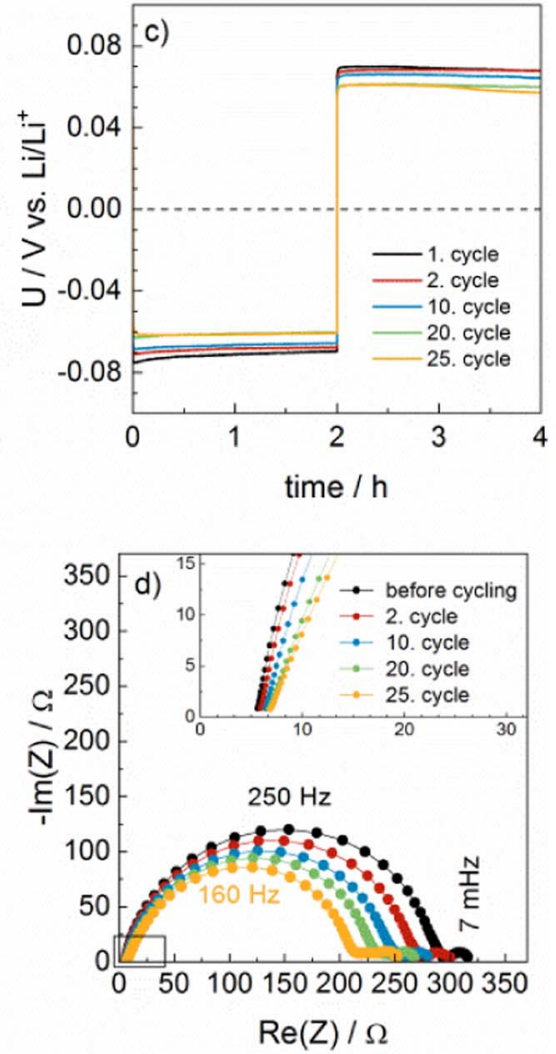

Figure 6. EIS analysis before and during stripping and deposition experiment on $\mathrm{Li} \| \mathrm{Li}$ cells: a) potential curves and b) impedance spectra measured for $\mathrm{Li}|| \mathrm{Li}$ cell with two Celgard 2320 separators. Inset shows magnification of the high frequency part of the spectra; comparison with c) potential curves and d) impedance spectra measured for $\mathrm{Li}|| \mathrm{Li}$ cell with NFC_20PO separator. Inset shows magnification of the high frequency part of the spectra. Before cycling, both cells were left for $24 \mathrm{~h}$ at $\mathrm{OCV}$ to stabilize. 
Celgard separator $(200 \Omega)$. This is also in good agreement with the initial reaction of hydroxide groups or water trapped in the cellulose and the lithium surface, as deduced from XPS measurements. Further cycling with Celgard separator reveals potential transients with two local maximums and two different potential plateaus. This shape is well known in the literature ${ }^{36,37}$ and can be associated with initial dissolution of lithium from formed dendrites and subsequent pitting and dissolution from bulk Li. The measured impedance spectra also verify that Li stripping and deposition with Celgard separator proceeds through formation of large quantities of HSAL. Namely, a decrease in the high frequency arc, corresponding to an increase in the surface area is visible, as well as transport difficulties through the porous and/or dead $\mathrm{Li}$ deposits. This is seen as an increase in the low frequency diffusion and high frequency migration contribution (compare the spectra on Figure $6 \mathrm{~b}$ and on its insert). Due to these effects, with further cycling, the overpotential of the cell with Celgard separator increases above the overpotential of the NFC separator cell (Figure 6a, $6 c)$. In the latter cell, both the potential transients and the impedance spectra measured are very stable (Figures $6 \mathrm{c}, 6 \mathrm{~d}$ ). The potential shows a uniform plateau, indicative of smooth deposition of Li beneath the SEI surface layer. With time, the overpotential slightly decreases, which we attribute to the formation of a few HSAL islands at points where SEI stability was poor (see Figure $4 \mathrm{c}$ ). By the $25^{\text {th }}$ cycle of stripping and deposition, spikes appear in the potential curves for the cell with Celgard separator, which are attributed to micro short circuits and indicate cell failure. In summary, EIS validates that when NFC separator is used, HSAL formation is hindered. Furthermore, with the use of cellulose based separator the stability of the cell regarding the tendency to exhibit short circuits is improved.

\section{Conclusions}

Nano-fibrillated cellulose has many outstanding properties such as large surface area, easy modification, biodegradability, good mechanical properties and chemical durability. Nano-fibrillated cellulose separator with tuneable thickness and porosity was successfully developed in this work. Compared to a commercial polyolefin separator, the NFC separator displays good wettability and better electrolyte uptake and, consequently, improves the $\mathrm{Li}-\mathrm{S}$ battery performance and rate capability. Moreover, due to unique morphology and interplay between lithium metal and cellulose, usage of NFC separator hinders formation of high surface area lithium (HSAL) and, in that way, reduces the degradation of lithium metal anode. These excellent properties make NFC separator promising battery material for next generation, high energy density lithium-sulfur and other lithium metal batteries.

\section{Acknowledgments}

This work was financially supported by the HELIS project funded from the European Union's Horizon 2020 research and innovation programme under grant agreement No 666221 and from the frame of Slovenian smart specialization programme CELLCYCLE under grant agreement C3330-16-529004. We thank Jernej Bobnar for SEM analysis of lithium samples and Helena Spreizer for contact angle measurements.

\section{ORCID}

Rémi Dedryvère (1D https://orcid.org/0000-0001-5125-640X

Robert Dominko (D) https://orcid.org/0000-0002-6673-4459

\section{References}

1. P. G. Bruce, S. A. Freunberger, L. J. Hardwick, and J.-M. Tarascon, Nat. Mater, 11, 19 (2012).

2. N. Choi et al., Angew. Chemie Int. Ed., 51, 9994 (2012).

3. X. Ji, K. T. Lee, and L. F. Nazar, Nat. Mater, 8, 500 (2009).

4. B. Zhang, X. Qin, G. R. Li, and X. P. Gao, Energy Environ. Sci., 3, 1531 (2010).

5. J. Schuster et al., Angew. Chemie Int. Ed., 51, 3591 (2012).

6. X. Liang et al., Nat. Commun., 6, 5682 (2015).

7. M. Cuisinier et al., Energy Environ. Sci., 7, 2697 (2014)

8. S. Drvarič Talian et al., Chem. Mater, 29, 10037 (2017)

9. S. Gu et al., Phys. Chem. Chem. Phys., 18, 29293 (2016).

10. N. Azimi et al., ACS Appl. Mater. Interfaces, 7, 9169 (2015)

11. N. Deng et al., J. Power Sources, 331, 132 (2016).

12. T. Placke, R. Kloepsch, S. Dühnen, and M. Winter, J. Solid State Electrochem., 21, 1939 (2017).

13. D. Lin, Y. Liu, and Y. Cui, Nat. Nanotechnol., 12, 194 (2017).

14. H. Lee, D. J. Lee, Y.-J. Kim, J. Park, and H. Kim, J. Power Sources, 284, 103 (2015).

15. A. Vizintin et al., Chem. Mater, 27, 7070 (2015).

16. X. Huang, J. Solid State Electrochem., 15, 649 (2011).

17. J. Zhang et al., Sci. Rep., 4, 3935 (2015).

18. F. Jiang, L. Yin, Q. Yu, C. Zhong, and J. Zhang, J. Power Sources, 279, 21 (2015).

19. B.-C. Yu, K. Park, J. Jang, and J. B. Goodenough, ACS Energy Lett., 1, 633 (2016).

20. R. Pan et al., Small, 14, 1704371 (2018).

21. H. Liao et al., J. Memb. Sci., 514, 332 (2016).

22. S.-J. Chun et al., J. Mater. Chem. A, 22, 16618 (2012).

23. Z. Wang et al., ACS Appl. Energy Mater., 1, 4341 (2018).

24. J.-H. Kim et al., Nanomat LiS batteries based on all fibrous cathode/separator assemblies and reinforced Li metal anodes: towards ultrahigh energy density and flexibility, Energy Environ. Sci. (2019).

25. J.-H. Kim et al., J. Power Sources, 242, 533 (2013).

26. M. Henriksson, L. A. Berglund, P. Isaksson, T. Lindström, and T. Nishino, Biomacromolecules, 9, 1579 (2008)

27. S. S. Zhang, J. Power Sources, 164, 351 (2007)

28. P. Onu, C. Vasile, S. Ciocîlteu, E. Iojoiu, and H. Darie, J. Anal. Appl. Pyrolysis, 49, 145 (1999).

29. H. Bockhorn, A. Hornung, U. Hornung, and D. Schawaller, J. Anal. Appl. Pyrolysis, 48, 93 (1999).

30. Q. Pang, X. Liang, C. Y. Kwok, and L. F. Nazar, Nat. Energy, 1, 16132 (2016).

31. D. Aurbach, E. Zinigrad, Y. Cohen, and H. Teller, Solid State Ionics, 148, 405 (2002).

32. V. Lapornik et al., J. Power Sources, 274, 1239 (2015).

33. D. Aurbach et al., J. Power Sources, 68, 91 (1997).

34. H. Cheng, C. Zhu, M. Lu, and Y. Yang, J. Power Sources, 173, 531 (2007).

35. Y. Guo, H. Li, and T. Zhai, Adv. Mater, 29, 1700007 (2017).

36. K.-H. Chen et al., J. Mater. Chem. A, 5, 11671 (2017)

37. K. N. Wood et al., ACS Cent. Sci., 2, 790 (2016). 